\section{Care home staff knowledge of oral care compared to best practice: a West of Scotland pilot study}

\author{
B. C. Young, ${ }^{1}$ C. A. Murray ${ }^{2}$ and J. Thomson ${ }^{3}$
}

IN BRIEF

- Standards of knowledge of oral care provision for the elderly within care homes in the West of Scotland has been identified as sub-optimal.

- Oral health educator training based upon the NHSOIS 'protocol for daily oral care' was demonstrated to improve knowledge and attitudes of surveyed staff.

- The explanatory nature of this study highlights a number of important areas for future research.

\begin{abstract}
Aim To evaluate care home $(\mathrm{N})$ staff knowledge of oral care provision for dependent older people in comparison to guidelines from NHS Quality Improvement Scotland (NHSOIS). This pilot study also aimed to identify barriers to delivering oral care and determine if oral health educator (OHE) training had an effect upon staff knowledge of oral care delivery. Setting This crosssectional analytic investigation was undertaken within the Greater Glasgow \&t Clyde area between 2005 and 2007. Methods From 33 care homes $(N), 28$ participated in data gathering through an interview schedule involving 109 staff. A 'knowledge check-list' founded upon daily oral care guidelines from the NHSOIS best practice statement (BPS) served as a template for knowledge assessment. An OHE undertook small group discussions related to the BPS in a sub-group of original participants and a second round of data was collected. Results The majority of surveyed staff $(n=86,79 \%)$ agreed that residents required assistance with oral care and placed oral care $(n=85,78 \%)$ in a moderate to high priority. However, only $57 \%$ of managers and $49 \%$ of nurses had received training in oral care provision. Most staff (79\% of managers, $85 \%$ of nurses) were unaware of the NHSQIS BPS. Deficiencies in knowledge were identified in several areas of the BPS. In particular, knowledge in the care of the natural dentition was inadequate. Between pre- and post-OHE training, the research suggests the following areas are liable to change: prioritisation given to oral care $(p=0.01)$, perceived competence $(p<0.0001)$ and confidence in providing oral care advice $(p<0.0001)$. Following OHE intervention, staff knowledge in oral care procedures compliant with best practice guidelines increased by $45 \%$. Conclusions Knowledge of oral health provision by those responsible for the care of home residents was deficient. An OHE training programme structured around the NHSOIS BPS demonstrated a measurable increase in levels of staff knowledge of oral care procedures.
\end{abstract}

\section{INTRODUCTION}

The 'elderly support ratio' can be defined as the total number of working age citizens within a population divided by the number of people at pensionable age. As the number of dependent elderly people continues to increase within the United Kingdom, ${ }^{1}$ the elderly support ratio is projected to decline from 3.35 in 2002 to below 2.2 by the 2050s. Currently, there are approximately 450,000 occupied places in residential and nursing

${ }^{1 *}$ Staff Grade in Restorative Dentistry, ${ }^{2}$ Clinical Senior Lecturer/Honorary Consultant in Restorative Dentistry, University of Glasgow Dental Hospital and School, Restorative Section, Level 6, 378 Sauchiehall Street, Glasgow, G2 3JZ; ${ }^{3}$ Lecturer in Marketing, Department of Marketing, University of Stirling, Stirling, FK9 4LA

${ }^{*}$ Correspondence to: Miss Beth Young

Email: beth_the_dentist@hotmail.com

\section{Omline article number E15}

Refereed Paper - accepted 12 March 2008

DOI: $10.1038 /$ sj.bdj.2008.894

${ }^{\oplus}$ British Dental Journal 2008; 205: E15 care homes and hospitals within the UK. ${ }^{1}$ To accommodate the increased care demands resulting from a projected rise in elderly population, availability of places in care homes and hospitals would need to increase by about $151 \%$ to approximately $1,130,000$ by $2051 .{ }^{1}$

It is recognised that people who utilise residential services place particular emphasis on certain features about the care and support they receive. Such features include being involved in decisions about their individual care requirements and being assisted by people who have received appropriate training. ${ }^{2}$ In Scotland, the Health Care Commission governs training requirements for care home staff and dictates the appropriate ratio of trained to untrained staff for the service. This is reviewed at regular intervals. Nurses must be registered with their regulatory body, The Nursing and Midwifery Council, and maintain their standards and registration every three years. Those with responsibility for management of care homes must be registered with the Scottish Social Services Council and comply with specified minimum training requirements. ${ }^{3}$ Care homes are required to provide new staff with a clearly defined training programme. Training plans should be reviewed annually for all staff and homes must ensure that all the necessary skills for practical care provision are covered during training. ${ }^{2}$

Elderly patients are likely to suffer several adverse oral conditions including xersostomia, increased caries risk, advanced toothwear, deficient levels of oral hygiene due to associated medical conditions, loss of natural teeth, compromised dento-alveolar bone support for removable prostheses and ongoing periodontal disease. ${ }^{4}$ Although a poor level of oral care is unlikely to be fatal, it 
can have a negative impact upon quality of life, the ability to masticate, communication and general personal comfort. ${ }^{5,6}$ Deficient oral care within care homes is a long-standing and widely recognised problem. ${ }^{7-9}$ It is also recognised that the quality of oral health education imparted by care home staff varies tremendously. ${ }^{10}$

Prior to publication of the best practice statement Working with dependent older people to achieve good oral health by NHS Quality Improvement Scotland (NHSQIS) in 2005, ${ }^{11}$ there were limited guidelines for this area of healthcare. However, the release of this document has bestowed care homes with a target for providing the best and achievable standard of oral care provision for Scotland's elderly population. The statement places emphasis on the principles of patient-centred care that is consistent, cohesive, fair and cost-effective. It aims to offer guidance in attaining and sustaining good practice. Care home staff are encouraged to work to their full potential, which NHSQIS hope will in turn stimulate learning and promote interdisciplinary team working. The statement is based upon an agreed set of values developed by the Scottish Gerodontology Community of Practice and is due for review in 2008 and every three years thereafter.

Since the Regulation of Care (Scotland) Act 2001 was introduced, ${ }^{12}$ nursing homes and care homes within Scotland are both legally classified as care homes. However, they are sometimes differentiated as care homes $(\mathrm{N})$ and care homes (R), respectively. Within Scotland, both provide nursing care where required. This is dependent upon their personal specifications and flexibility of services. For the purpose of this paper, participating care homes have been referred to as 'care home $(\mathrm{N})$ '.

This cross-sectional analytic survey primarily intended to determine the degree of dissemination of the NHSQIS best practice statement (BPS) to care homes $(\mathrm{N})$ within the Greater Glasgow ct Clyde area. In addition, the attitude, confidence and current training levels of care home (N) staff in the provision of oral healthcare were investigated. Furthermore, knowledge levels of care home

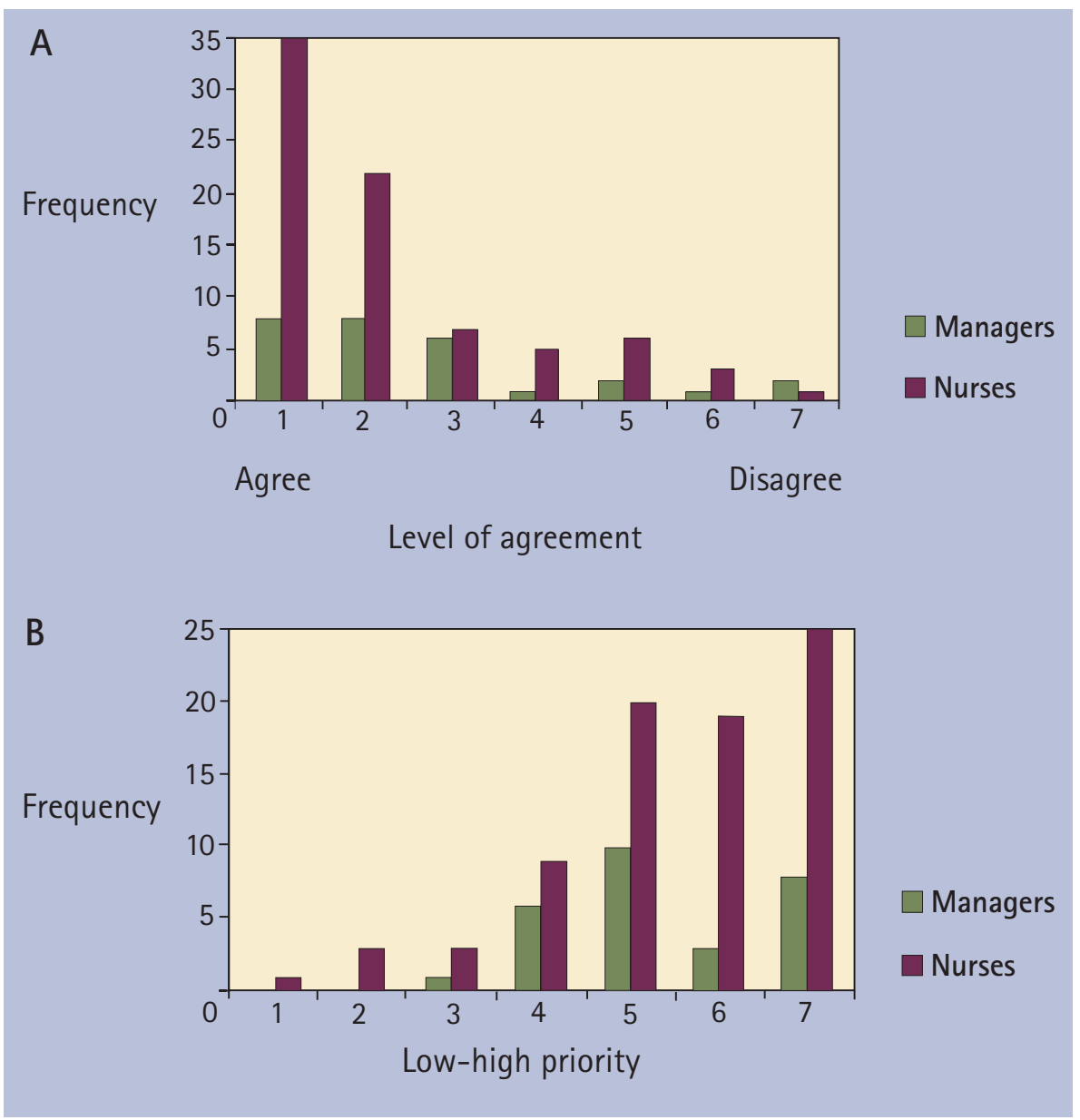

Fig. 1 Requirements for the provision of oral care within nursing homes. A) staff opinions that nursing home residents desired assistance with oral hygiene procedures, B) priority given to oral care by staff in comparison to oveall healthcare duties

(N) managers and nursing staff in oral care measures were assessed and compared to the NHSQIS BPS. Finally, the study evaluated if staff training from oral health educator (OHE) training had a positive effect upon care home $(\mathrm{N})$ staff knowledge pertaining to the NHSQIS BPS.

\section{METHODS}

\section{Initial assessment of attitudes to and knowledge of oral care provision}

Thirty-three care homes (N) within the Greater Glasgow \&t Clyde region were randomly selected by an internet search engine. A letter was posted to these homes (Appendix 1) inviting staff to participate in this pilot study. The letter introduced the project and established arrangements for administering an interview schedule with three members of nursing care staff and the manager of each home. To ensure that a valid data comparison could be made between participating staff, only registered nurses, as opposed to carers from a broad variety of occupations, were invited to participate. At this stage, there was deliberately no mention of the NHSQIS best practice statement.

Interview schedules were administered over the telephone (see Appendix 2) by a researcher. This methodology was selected to ensure that good quality responses were obtained whilst overcoming some of the pitfalls of postal questionnaires, particularly poor response rates. In order to standardise techniques, the four researchers responsible for data collection received calibrated training on the interview technique prior to their commencement. To facilitate measurement of various dimensions of attitudes towards oral healthcare provision and levels of staff training, the first two components of the interview schedule were designed using Likert scales. The third section adopted an open-ended approach whereby staff were invited to volunteer 
information in response to questions rather than being offered a scaled response. These questions were formulated to enable a comparison between staff knowledge and NHSQIS BPS.

A 'knowledge check-list' constructed around the BPS protocol for daily oral care (Appendix 3) was used as the template for knowledge assessment. The interview schedule and 'knowledge check-list' were designed to allow accurate comparisons of pre- and post-education knowledge following training from an oral health educator (OHE). One mark was allocated per correct point mentioned from the NHSQIS 'protocol for daily oral care'. The total 'score' for each participant was then calculated and averaged for all staff involved and then assigned a percentage. Registered nurses on duty at the time of the telephone call were invited to volunteer for interview.

\section{Sub-group assessment following OHE intervention}

Following completion of the first round of questionnaires, an OHE undertook small group training sessions with the three members of the nursing staff and a home manager within a subgroup of care homes (N) from the South Glasgow area. Participants in this stage of the research form a convenience sample of $n=24$. Training was centred upon providing knowledge of the protocol for daily oral care within the NHSQIS BPS. Training was provided by the same OHE for all groups and each training session lasted three hours.

From this sub-group, a second round of anonymised data was collected within one month of OHE training by the same members of research staff involved in data collection from the original care home $(\mathrm{N})$ group. Statistical analysis was performed on collected data using Excel (Microsoft Corporation, USA) and SPSS (SPSS Inc., Chicago, USA).

\section{RESULTS}

\section{A. Initial assessment of attitudes towards and knowledge of oral care provision}

Of the 33 care homes $(\mathrm{N})$ invited to take part in the study, 28 participated in data gathering. Data was collected from a

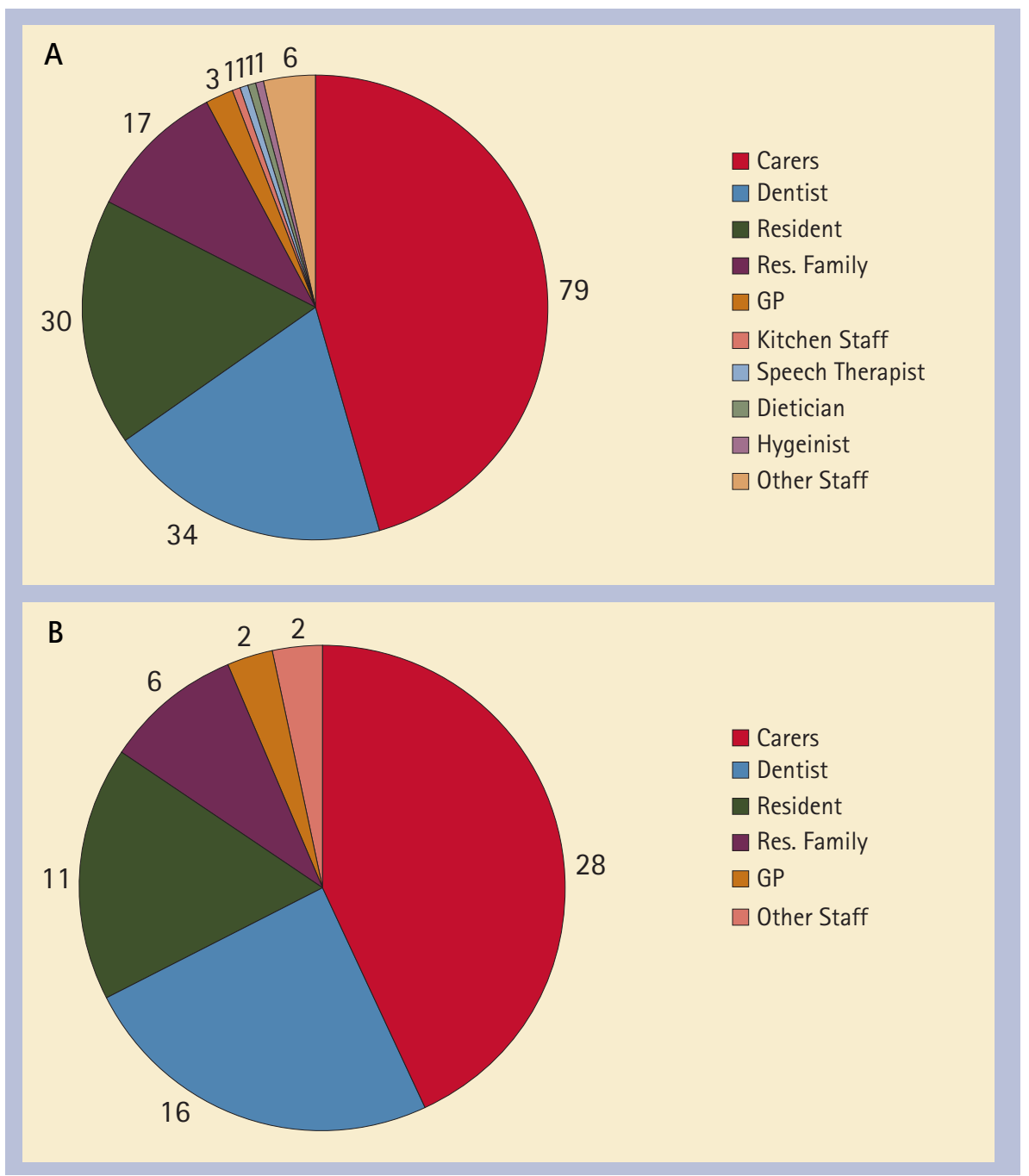

Fig. 2 Responsibility of non-nursing staff in delivery of oral care. A) members of the care team that nurses held primarily responsible for oral care provision (excluding nurses themselves), B) members of the care team nursing home managers viewed primarily responsible for oral care (other than nurses)

total of 109 staff in these care homes (N). Non-participating homes advised that they were too busy to be involved.

\section{Requirements for the provision of oral care}

When asked to express level of agreement with the statement 'All residents in your care desire assistance with oral hygiene procedures', where 1 represents strongly agree, and 7 represents strongly disagree (Fig. 1A), the majority of those surveyed $(\mathrm{n}=86)$ agreed that all their residents required assistance with oral care (mean score 2.71 [SD $=1.802$ ] for managers and 2.25 [SD = 1.563] for nurses). The majority of surveyed staff placed oral care in a moderate to high priority category (n = 85) when compared to overall healthcare requirements (mean score 5.39 [SD $=1.227$ for managers and 5.51 [SD = 1.432] for nurses, Fig. 1B).
Responsibility of healthcare givers in delivering oral care

Nurses recruited to this study were differentiated from carers and defined as individuals registered with The Nursing and Midwifery Council. When asked which members of the care home $(\mathrm{N})$ team, excluding nursing personnel, were primarily responsible for providing oral care (Fig. 2A), 45\% ( $\mathrm{n}=79)$ of nurses stated this was the responsibility of carers. Other most frequent responses for oral care provision included the dentist $(19 \%, \mathrm{n}=34)$ and the resident $(17 \%$, $\mathrm{n}=30)$. Care home $(\mathrm{N})$ managers also most commonly named carers as having primary responsibility for the oral care provision of residents $(43 \%, n=28$, Fig. 2B). Similar to the surveyed nurses, 25\% $(n=16)$ of managers considered dentists to be responsible for the oral care of residents and 17\% $(n=11)$ considered 
the resident responsible for their own oral care.

\section{Training in oral health measures}

Of those surveyed, 57\% ( $n=16)$ of managers and $49 \%(n=39)$ of nurses stated they had been trained in delivering oral care. The majority of managers indicated they had received their training in the form of undergraduate lectures (38\%, n $=9$ ). Other forms of training included undergraduate practical lessons (25\%, n $=6)$ or from a dentist $(13 \%, \mathrm{n}=3$, Fig. 3A). Of the nurses surveyed, 29\% ( $\mathrm{n}=$ 19) had received training from undergraduate lectures, 23\% ( $\mathrm{n}=15)$ from undergraduate practical lessons, 22\% (n $=15)$ in-house and 12\% (n=8) via an oral health educator (Fig. 3B).

\section{Potential barriers to oral care training}

The most common barriers to care home (N) managers not receiving appropriate training in oral care provision were the lack of availability for this in the home in which they worked $(85 \%, \mathrm{n}=11)$ or that oral care had not been covered in their university or college course (15\%, $n=2$ ) (Fig. 3C). Of those nurses who had not received formal training in oral healthcare, the most frequently cited reasons included training not being available in their care home (N) $(74 \%, n=36)$ or that oral care had not been covered within the college/university curriculum $(20 \%, n=10)$. Other barriers included absence from work on training days $(4 \%, n=2)$ or being too busy for training (2\%, $n=1$ ) (Fig. 3D).

\section{Potential barriers to providing oral care}

Most of the enrolled managers (82\%, $n$ $=23)$ and nurses $(76 \%, \mathrm{n}=61)$ agreed or very much agreed with the statement that lack of resident co-operation was a barrier to providing a suitable standard of oral care. The majority of surveyed managers $(79 \%, \mathrm{n}=22)$ and nurses (86\%, $\mathrm{n}=69$ ) stated they strongly agreed/agreed that sufficient time was available to perform oral care measures for care home $(\mathrm{N})$ residents.

\section{Dissemination of NHSOIS best practice statement}

Dissemination of the NHSQIS BPS was
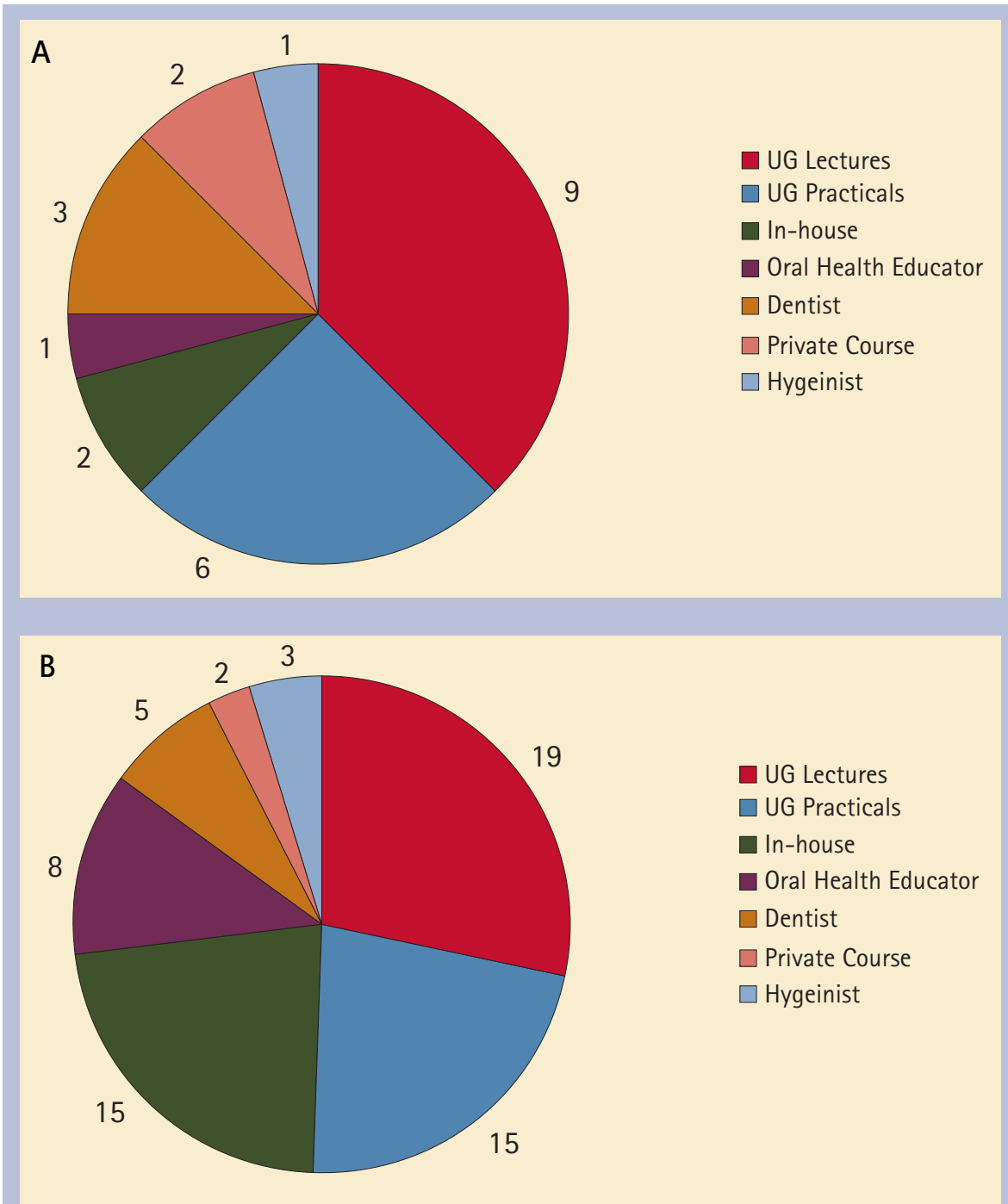

C

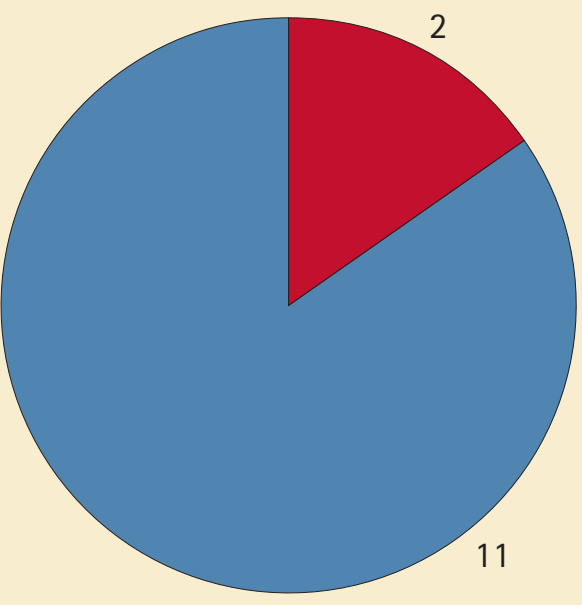

$\mathrm{N} / \mathrm{C}$ College

None Available in Home

Fig. 3 Training in relation to oral care provision. A) methods by which managers had received training in oral care provision, B) methods by which nurses had been trained in provision of oral care, C) reasons for lack of training in oral care given by surveyed managers

found to be very low amongst participating staff. The vast majority of surveyed managers $(79 \%, \mathrm{n}=22)$ and nurses $(85 \%, n=67)$ were unaware of the NHSQIS best practice statement (Fig. 3E).

\section{Knowledge of oral health care}

When compared to the knowledge checklist compiled from the NHSQIS 'protocol for daily oral care', the average overall score for knowledge of oral care 


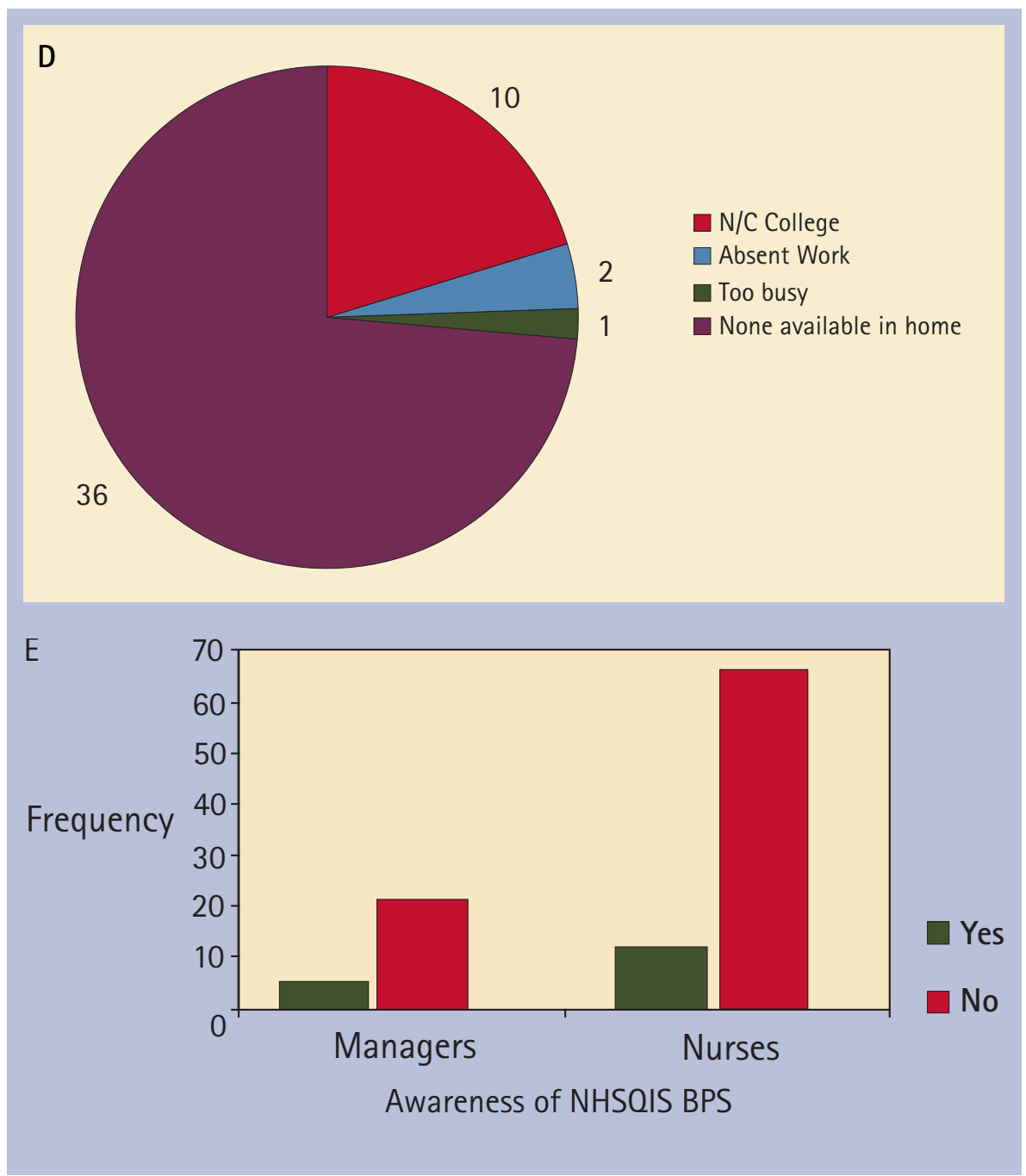

Fig. 3 Training in relation to oral care provision. D) reasons for lack of training in the provision of oral care by nurses who had not received previous training, E) home manager and nurse awareness of NHSOIS BPS

procedures was only 30\% for managers and for nurses, 37\%. Particular shortfalls were identified in knowledge of denture care (Table $1 \mathrm{~A})$, care of natural teeth (Table 1B), care of oral mucosa (Table 1C) and care of a dry mouth (Table 1D).

Group statistics performed on collected data did not reveal any statistically significant differences between nursing or manager groups for responses regarding attitudes, knowledge, competence or confidence in oral care procedures. As such, a manager with a poor knowledge or attitude towards oral care was likely to have nurses with a similarly poor knowledge and attitude within their team.

\section{B. Sub-group assessment following OHE intervention}

Following data collection from all study participants, sub-group data analysis $(\mathrm{p}=0.01$; mean rating pre-OHE 5.47, $\mathrm{SD}=1.99$; mean rating post-OHE 6.30, $\mathrm{SD}=0.77$; Fig. 4A), self-competence assessment ( $p<0.0001$; mean pre-OHE involvement score $2.43, \mathrm{SD}=1.52$; mean post-OHE 1.56, SD = 0.44; Fig. 4B) and confidence advising other staff in oral hygiene procedures ( $p<0.0001$; mean pre-OHE score $2.43, \mathrm{SD}=1.52$; mean postOHE training $1.56, \mathrm{SD}=0.44$; Fig. $4 \mathrm{C}$ ).

\section{Knowledge of oral healthcare provision}

Prior to OHE training, the overall score for staff knowledge of oral care procedures was a mean of $42 \%$. Following OHE training, oral health care knowledge scoring rose to $82 \%$. This represented a $41 \%$ increase in correct responses in oral care provision. After OHE intervention, particular improvements were identified in aspects of oral mucosa care (Table $2 \mathrm{~A}$ ), care of a dry mouth (Table $2 \mathrm{~B}$ ), care of resident's natural teeth (Table $2 \mathrm{C}$ ) and care of edentulous residents with removable prostheses (Table 2D).

\section{DISCUSSION}

Not only is good oral health important for quality of life, but it is also associated with general health. Poor oral care is a widely recognised problem and if left unattended, may have wider health implications. These include predisposition to conditions such as staphylococcal mucositis $^{13}$ or aspiration pneumonia. ${ }^{14,15}$ Such adverse ailments are most commonly observed in debilitated patients, for example elderly residents in care homes. Furthermore, oral discomfort has been associated with poor nutritional status in incapacitated patients. ${ }^{16} \mathrm{Popu}-$ lation projections since the early 1980s have identified that the number of older people retaining at least some of their natural teeth will continue to increase ${ }^{17}$ and that the population is ageing. ${ }^{1}$ This combination of factors will likely place increased demands upon the delivery of oral healthcare by care home staff.

There are strict legislative controls implemented by the Care Commission Scotland and the Scottish Social Services Council that govern care home $(\mathrm{N})$ staff. The Care Commission Scotland is required to regularly inspect and monitor care homes $(\mathrm{N})$ with respect to 
levels of training and the specified ratio of trained to untrained staff. Care homes (N) invited to participate in this study were required to employ at least one fully-trained and qualified nurse to provide 24 hour on-call cover. In an attempt to ensure a standardised baseline level of education and knowledge across survey participants, only registered nursing staff and registered managers were approached to participate in the study. The study participation rate was high within both the initial survey group and sub-group population that received training from an OHE. 85\% of the contacted nursing homes participated in the survey. Furthermore, 96\% of those staff who undertook educational training in oral care procedures given by the OHE were available for the second round of data collection. The five care homes $(\mathrm{N})$ who declined to participate cited insufficient time as the underlying issue. A lack of time was also mentioned as a factor preventing subsequent attendance at training events. Nevertheless, a shortage of time was not identified by surveyed care home $(\mathrm{N})$ staff as a factor precluding the delivery of oral care for their home residents.

Assessment of surveyed participants' knowledge of oral care provision in comparison to knowledge check points contained within the NHSQIS 'protocol for daily oral care' revealed major deficiencies in current knowledge levels in the delivery of oral care. It was therefore not surprising to find that dissemination of the BPS amongst participating staff was very low, with the majority of managers (79\%) and nurses (85\%) unaware of the document. The NHSQIS BPS places emphasis on promoting interdisciplinary team working. Other than qualified nurses, carers were generally identified as those predominantly responsible for the delivery of oral care to home residents. However, whether responsibility for oral care should be delegated to 'kitchen staff', as suggested by one staff member, is somewhat debatable.

With an aging population retaining more of their dentition, it is concerning to establish that the standard of staff knowledge in care of the natural dentition was sub-optimal. Of those staff surveyed prior to OHE training, only

Table 1 Knowledge of oral care compared to NHSOIS 'protocol for daily oral care'. A) Care of the edentulous person with dentures, B) Care of natural teeth, C) Care of the oral mucosa, D) Care of the person with a dry mouth

A

\begin{tabular}{|l|l|l|l|}
\hline Denture care & Managers & Nurses & Total \\
\hline Denture marking & $0(0 \%)$ & $1(1 \%)$ & $1(5 \%)$ \\
\hline Dentures removed at night & $23(82 \%)$ & $77(96 \%)$ & $100(92 \%)$ \\
\hline Acrylic dentures in NaHCl & $22(79 \%)$ & $68(85 \%)$ & $90(83 \%)$ \\
\hline Clean using individual brush \& running water & $20(71 \%)$ & $54(68 \%)$ & $74(68 \%)$ \\
\hline Rinsed after meals & $7(25 \%)$ & $40(50 \%)$ & $47(43 \%)$ \\
\hline Fixative if required & $1(4 \%)$ & $2(3 \%)$ & $3(3 \%)$ \\
\hline Fixative cleaned off if required & $0(0 \%)$ & $2(3 \%)$ & $2(2 \%)$ \\
\hline $\begin{array}{l}\text { Knowledge of difference between acrylic } \\
\text { and CoCr care }\end{array}$ & $0(0 \%)$ & $2(3 \%)$ & $2(2 \%)$ \\
\hline $\begin{array}{l}\text { CoCr dentures to be soaked in chlorhexidine } \\
\text { not NaHCl }\end{array}$ & $0(0 \%)$ & $3(4 \%)$ & $3(3 \%)$ \\
\hline Overall & $73 / 252(29 \%)$ & $249 / 720(35 \%)$ & $322 / 972(33 \%)$ \\
\hline
\end{tabular}

B

\begin{tabular}{|l|l|l|l|}
\hline Care of natural teeth & Managers & Nurses & Total \\
\hline Cleaned twice daily and after meals & $16(57 \%)$ & $2(3 \%)$ & $18(17 \%)$ \\
\hline Fluoride toothpaste & $5(18 \%)$ & $20(25 \%)$ & $25(23 \%)$ \\
\hline Soft toothbrush & $21(75 \%)$ & $50(63 \%)$ & $71(65 \%)$ \\
\hline Corsodyl if required & $1(4 \%)$ & $9(11 \%)$ & $8(7 \%)$ \\
\hline Powered toothbrush if preferred & $2(7 \%)$ & $2(3 \%)$ & $4(4 \%)$ \\
\hline Overall & $45 / 140(32 \%)$ & $83 / 400(21 \%)$ & $128 / 540(24 \%)$ \\
\hline C & & &
\end{tabular}

C

\begin{tabular}{|l|l|l|l}
\hline Care of oral mucosa & Managers & Nurses & Total \\
\hline Inspect in good light & $3(11 \%)$ & $10(13 \%)$ & $13(12 \%)$ \\
\hline Report & $11(40 \%)$ & $32(40 \%)$ & $43(39 \%)$ \\
\hline Clean & $10(36 \%)$ & $32(40 \%)$ & $42(39 \%)$ \\
\hline Overall & $24 / 84(31 \%)$ & $71 / 240(31 \%)$ & $98 / 324(30 \%)$ \\
\hline D & & & \\
\hline Care of dry mouth & Managers & Nurses & Total \\
\hline Sips of water/saliva substitute & $28(100 \%)$ & $67(83.7 \%)$ & $75(69 \%)$ \\
\hline High fluoride toothpaste & $1(4 \%)$ & $0(0 \%)$ & $1(1 \%)$ \\
\hline Overall & $29 / 56(52 \%)$ & $67 / 160(42 \%)$ & $96 / 216(32 \%)$ \\
\hline
\end{tabular}

$52 \%$ knew how to correctly care for a dry mouth. Of particular note, $87.9 \%$ of care home $(\mathrm{N})$ staff $(\mathrm{n}=20)$ advised that lemon and glycerine should be used to alleviate symptoms of xerostomia. This would likely lead to severe erosion in a dentate patient suffering from xerostomia. Furthermore, the use of fluoride toothpaste, an essential component in the prevention of dental caries, was mentioned by only one member of surveyed staff $(4 \%)$ prior to OHE involvement. Following OHE training, recommendation on the use of a fluoride toothpaste rose to $100 \%(n=23)$. Potential use of a powered as opposed to a manual toothbrush for those with poor manual dexterity was mentioned by only two of 
Table 2 Sub-group knowledge of oral care compared to NHSOIS 'protocol for daily oral care'. A) care of the oral mucosa, B) care of the person with a dry mouth C) care of natural teeth, D) care of the edentulous person with dentures

\section{A}

\begin{tabular}{|l|l|l|l|}
\hline Care of oral mucosa & Before & After & Difference \\
\hline Inspect in good light & $7(30 \%)$ & $20(87 \%)$ & $13(57 \%)$ \\
\hline Report & $5(22 \%)$ & $20(87 \%)$ & $15(65 \%)$ \\
\hline Clean & $11(48 \%)$ & $23(100 \%)$ & $12(52 \%)$ \\
\hline Total & $23 / 69(33 \%)$ & $63 / 69(91 \%)$ & $40(58 \%)$ \\
\hline
\end{tabular}

\section{B}

\begin{tabular}{|l|l|l|l|}
\hline Care of dry mouth & Before & After & Difference \\
\hline Sips of water/saliva substitute & $23(100 \%)$ & $23(100 \%)$ & $0(0 \%)$ \\
\hline High fluoride toothpaste & $1(4 \%)$ & $23(100 \%)$ & $22(96 \%)$ \\
\hline Total & $24 / 46(52 \%)$ & $46 / 46(100 \%)$ & $22(48 \%)$ \\
\hline C
\end{tabular}

C

\begin{tabular}{|l|l|l|l|}
\hline Care of natural teeth & Before & After & Difference \\
\hline Cleaned twice daily and after meals & $6(26 \%)$ & $11(48 \%)$ & $5(22 \%)$ \\
\hline Fluoride toothpaste & $1(4 \%)$ & $23(100 \%)$ & $22(96 \%)$ \\
\hline Soft toothbrush & $22(96 \%)$ & $23(100 \%)$ & $1(4 \%)$ \\
\hline Corsodyl if required & $4(17 \%)$ & $22(96 \%)$ & $18(78 \%)$ \\
\hline Powered toothbrush if preferred & $2(9 \%)$ & $8(35 \%)$ & $6(26 \%)$ \\
\hline Total & $35 / 115(30 \%)$ & $87 / 115(76 \%)$ & $52(45 \%)$ \\
\hline D & & &
\end{tabular}

D

\begin{tabular}{|l|l|l|l|}
\hline Denture care & Before & After & Difference \\
\hline Denture marking & $0(0 \%)$ & $0(0 \%)$ & $0(0 \%)$ \\
\hline Dentures removed at night & $22(96 \%)$ & $23(100 \%)$ & $1(4 \%)$ \\
\hline Acrylic dentures in $\mathrm{NaHCl}$ & $19(83 \%)$ & $23(100 \%)$ & $4(17 \%)$ \\
\hline Clean using individual brush \& running water & $22(96 \%)$ & $23(100 \%)$ & $1(4 \%)$ \\
\hline Rinsed after meals & $5(22 \%)$ & $20(87 \%)$ & $15(65 \%)$ \\
\hline Fixative if required & $6(26 \%)$ & $18(78.3 \%)$ & $12(52 \%)$ \\
\hline Fixative cleaned off if required & $1(4 \%)$ & $3(13 \%)$ & $2(9 \%)$ \\
\hline $\begin{array}{l}\text { Knowledge of difference between acrylic and } \\
\text { CoCr care }\end{array}$ & $0(0 \%)$ & $22(96 \%)$ & $22(96 \%)$ \\
\hline $\begin{array}{l}\text { CoCr dentures to be soaked in chlorhexidine } \\
\text { not NaHCl }\end{array}$ & $0(0 \%)$ & $17(74 \%)$ & $17(74 \%)$ \\
\hline \begin{tabular}{l} 
Total \\
\hline
\end{tabular} & $75 / 207(36 \%)$ & $149 / 207(72 \%)$ & $74(36 \%)$ \\
\hline
\end{tabular}

the surveyed staff (8.7\%). However, it is somewhat disconcerting that following OHE intervention this figure rose to only eight $(34.8 \%)$.

Although the majority of staff recognised the requirement to clean dentures, clear deficiencies were identified in denture care within surveyed care homes (N). Denture marking has several advantages for the denture wearer. Most fundamental of these is denture identification, which facilitates the distinction of dentures between different wearers. This is particularly applicable for subjects in a care home environment. Denture identification marking would also enable the individual's dentures to be chronologically categorised according to the time of construction. This would be helpful in both primary and secondary care settings, particularly during clinical assessment when considering denture replacement. ${ }^{18}$ However, care home (N) staff were unaware that this was one of the key guidelines in the NHSQIS BPS. Within the BPS, a section relating to care of the edentulous patient with dentures expressly states that staff should "ensure that dentures are marked with the person's name (eg “Identure” Denture Marking System, Geri Incorporated)'. Of further concern, this lack of knowledge was evident not only prior to but also subsequent to OHE intervention.

Healthcare and management staff involved in this study rated oral care as a high priority. In contrast, their displayed knowledge in the provision of oral care was revealed to be severely deficient. Not only is training in oral care both desired and required by care staff, 7 it has also been demonstrated to be effective in a number of studies. ${ }^{19-23}$ Indeed, the important role that education and training in oral healthcare has upon improving attitudes and participants' ability to undertake tasks such as toothbrushing and increasing knowledge levels has previously been illustrated. ${ }^{24}$ It was therefore surprising that less than $50 \%$ of interviewed nurses had received formal training in oral care provision. Furthermore, of staff members that had received formal training, education in oral care measures had taken place as many as 40 years previously. A similar lack of emphasis on oral healthcare education during nurse training has been described in countries such as the USA and Sweden. ${ }^{24,25}$ In the care home (N) staff subgroup that received OHEbased training, knowledge, attitude and confidence in providing and advising on oral care were significantly improved. Moreover, feedback from those within the trained subgroup was very positive. Many staff stated they hoped such training in oral care would become available on a wider and more regular basis. This reinforces the requirement to ensure the provision of adequate training for all care home staff, particularly if homes are to comply with Personal Social Service's 
National Occupational Standards.

Our data support previous research identifying the positive benefits that OHEs have upon improving knowledge of nursing personnel in the provision of oral healthcare within care facilities. ${ }^{20}$ Indeed, these positive findings are in agreement with outcomes following the utilisation of oral health educators within other parts of the UK. ${ }^{26}$ Nevertheless, current evidence as to whether OHE training of patients or staff significantly enhances the oral care of population groups in the long-term is weak. Indeed, a recent study has demonstrated little meaningful clinical improvement in oral health of residents in long-term care facilities following nurse educator training. ${ }^{27}$ Our research was undertaken as a pilot study and hence only determined the effect of oral health education on a relatively small number of individual staff members. Further research is required to determine the long-term efficacy of oral health education based upon the best practice statement as a public health approach.

\section{CONCLUSION}

This investigation has identified that there continues to be inadequate training of staff within care homes $(\mathrm{N})$ in the provision of appropriate oral care to the elderly population utilising such facilities. There was poor dissemination of the NHSQIS best practice statement Working with dependent older people to achieve good oral health, and a lack of knowledge towards the content of this document. Of importance, this study has demonstrated the positive impact that an oral health educator has upon staff attitudes towards, and knowledge of, effective oral care procedures. This evidence may help form a basis for the delivery of future training to care home staff and should be considered when planning induction programmes for new care home staff members and staff training review meetings. The NHSQIS BPS has been specifically developed to provide an achievable basis for good practice in oral health measures. Inclusion of the practices described in the 'protocol for daily oral care' in resident care plans and staff training is designed to ensure the provision of a more consistent and cohesive

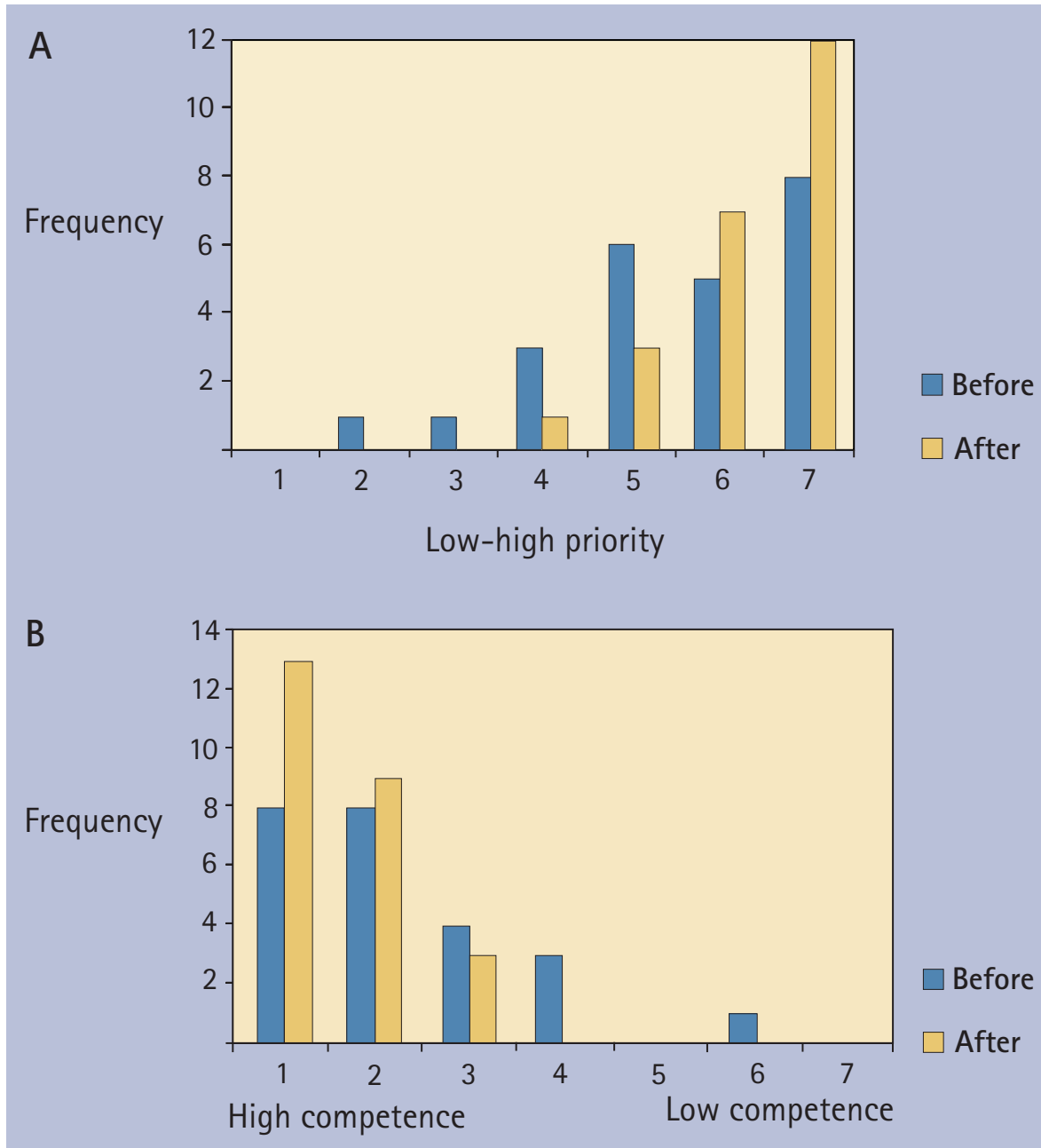

Competence

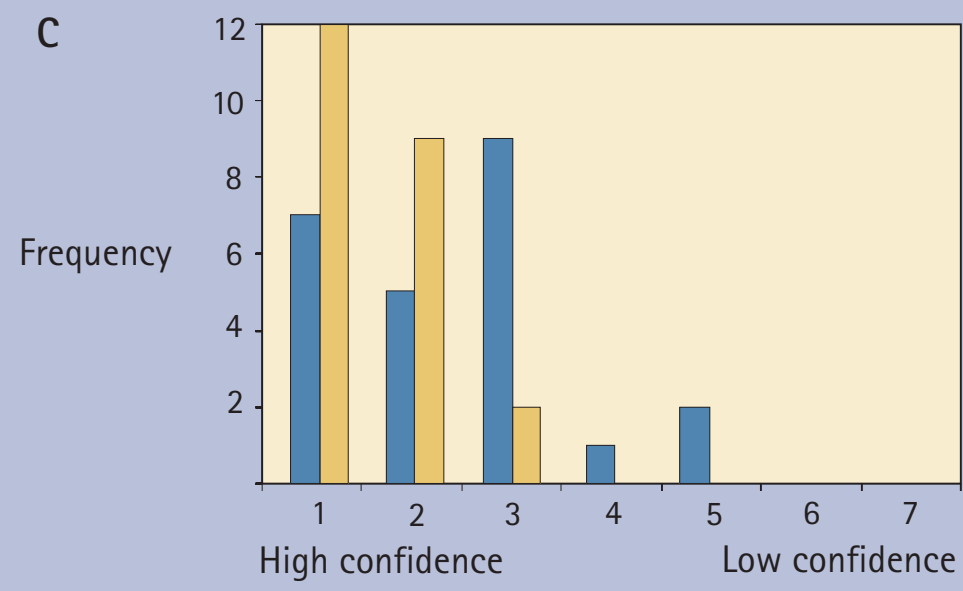

$\square$ Before $\square$ After

Confidence

Fig. 4 Effect of oral health educator (OHE) intervention in nursing home sub-group. A) priority given towards oral care by staff before and after OHE intervention, B) perceived levels of staff competence in provision of oral care measures to residents, C) perceived levels of staff confidence in giving advice on oral care to other healthcare staff (results are displayed as combined totals of nurses and managers pre- and post-OHE intervention)

level of care. Based on the findings of this pilot study, a training programme structured around these guidelines produces a measurable increase in levels of staff knowledge of oral care procedures. However, the long-term effect of 
OHE training of care staff upon the clinical status of elderly residents remains to be established.

The authors would like to thank Ray MacAndrew, Tara Dunseith and Sheila Hunter for help in setting up the study. The contributions from Nicola Cross, Laura Colgan and Pamela Yule in data collection are greatly appreciated. The authors also thank Eilidh MacRae for assistance in proof reading the manuscript and Petrina Sweeney for her helpful feedback.

1. Wittenberg R, Comas-Herrera A, Pickard L, Hancock R. Future demand for long-term care in the UK: a summary of projections of long-term care finance for older people to 2051. York: Joseph Rowntree Foundation, 2004.

2. Scottish Executive. National care standards - care homes for older people. Edinburgh: Scottish Executive, 2005. ISBN: 0-7559-4536-0.

3. Scottish Social Services Council. eBulletin, July 2007. Dundee: Scottish Social Services Council, 2007. http://www.sssc.uk.com/nr/rdonlyres/ 56b6574f-646b-4bc1-9674-7753e0b31166/0/ july2007ebulletin.pdf.

4. Christensen G J. Providing oral care for the aging patient. J Am Dent Assoc 2007; 138: 239-242.

5. McGrath C, Bedi R. The importance of oral health to older people's quality of life. Gerodontology 1999; 16: 15-20.

6. Paulsson G, Wardh I, Andersson P, Öhrn K. Comparison of oral health assessments between nursing staff and patients on medical wards. Eur J Cancer Care 2008; 17: 49-55.

7. Sweeney M P, Williams C, Kennedy C, Macpherson $L M$, Turner S, Bagg J. Oral health care and status of elderly care home residents in Glasgow. Community Dent Health 2007; 24: 37-42.

8. Preston A J, Kearns A, Barber M W, Gosney M A. The knowledge of healthcare professionals regarding elderly persons' oral care. Br Dent J 2006; 201: 293-295.

9. MacEntee M I. Oral care for successful aging in long-term care. J Public Health Dent 2000; 60: 326-329

10. Mynors-Wallis D M J Davis D M. An assessment of the oral health knowledge and recall after a dental talk amongst nurses working with elderly patients: a pilot study. Gerodontology 2004; 21: 201-204.

11. NHS Quality Improvement Scotland. Working with dependent older people to achieve good oral health. Edinburgh: NHS Quality Improvement Scotland, 2005. ISBN 1-84404-292-8.

12. The Scottish Parliament. Regulation of Care (Scotland) Act 2001. Edinburgh: The Stationery Office, 2001. ISBN 0-10-5 90018-4.

13. Smith A J, Jackson M S, Bagg J. The ecology of Staphylococcus species in the oral cavity. J Med Microbiol 2001; 50: 940-946.

14. Abe S, Ishihara K, Adachi M, Okuda K. Oral hygiene evaluation for effective oral care in preventing pneumonia in dentate elderly. Arch Gerontol Geriatr 2006; 43: 53-64

15. Azarpazhooh A, Leake J L. Systematic review of the association between respiratory diseases and oral health. J Periodontol 2006; 77: 1465-1482.

16. Soini H, Muurinen S, Routasalo P. Oral and nutritional status - is the MNA ${ }^{\circledR}$ a useful tool for dental clinics. J Nutr Health Aging 2006; 10: 495-499.

17. Nuttall N M, Davies J A. The frequency of denta attendance of Scottish dentate adults between 1978 and 1988. BrDent J 1991; 171: 161-165.

18. Murray C A, Boyd PT, Young B C, Dhar S, Dickson $M$, Currie J N W. A survey of denture identification marking within the United Kingdom. Br Dent $J$ 2007; 203: E24.

19. Nicol R, Sweeney M P McHugh E S, Bagg J. Effectiveness of health care worker training on the oral health of elderly residents of nursing homes. Community Dent Oral Epidemiol 2005; 33: 115-124.

20. Paulsson G, Söderfeldt B, Fridlund B, Nederfors T. Recall of oral health education programme by nursing personnel in special housing facilities for the elderly. J Gerodonto/ 2001; 18: 7-14.

21. Wyatt C C, So F H, Williams P M, Mithani A, Zed C M, Yen E H. The development, implementation, utilization and outcomes of a comprehensive dental program for older adults residing in long-term care facilities. J Can Dent Assoc 2006; 72: 419.

22. Chapman A, Copenstake S J, Duncan K. An oral health education programme based on the National Curriculum. Int J Paediatr Dent 2006; 16: 40-44.

23. Kay E J, Locker D. Is dental health education effective? A systematic review of current evidence. Community Dent Oral Epidemiol 1996; 24: 231-235.

24. Schmidt S M, Leach M, Nicolaci A B, Sutton B R, O'Donnell J P. The dental health educator and programs for institutions with persons who are mentally retarded. Spec Care Dentist 1981; 1: 174-178.

25. Paulsson G, Nederfors T, Fridlund B. Conceptions of oral health among nurse managers. A qualitative analysis. J Nurs Manag 1999; 7: 299-306.

26. Frenkel H, Harvey I, Newcombe R G. Improving oral health in institutionalised elderly people by educating caregivers: a randomised controlled trial. Community Dent Oral Epidemiol 2001; 29: 289-297.

27. MacEntee M I, Wyatt C C L, Beattie B L et al. Provision of mouth-care in long-term care facilities: an educational trial. Community Dent Oral Epidemiol 2007; 35: 25-34.

\section{Appendix 1}

\section{Community Dental Department}

Govanhill Health Centre

233 Calder Street

Glasgow

G42 7DR

Enquiries to:

Dr Beth Young

Telephone: 01415318331

E-mail: beth_the_dentist@hotmail.com

Dear Sir/Madam,

I am planning to carry out a survey investigating attitudes and level of knowledge of staff in long stay care homes, with regard to oral care of residents.

This research aims to determine the attitudes of nursing home managers and staff to oral care, as well as current levels of training, confidence and knowledge.

A questionnaire has been designed for this purpose.

It is hoped that the information gained from this exercise will help provide a basis for future research, training and guideline development.

I will telephone in the next week to organise a time for telephone interviews with the care home Manager and 3 members of the Nursing staff. The questionnaire should take only a few minutes for each person to complete and will be entirely confidential. Your help with this project would be much appreciated. If you have any questions prior to my call, I can be contacted as above.

Yours Sincerely,

Dr. Beth Young

GPT Community Dental Officer 
Appendix 2 Questionnaire

\section{Attitudes}

1) With regards to the daily duties required of nursing care staff, please indicate the level of priority given to oral care: Please indicate the number which applies:

$\begin{array}{llllllllll}\text { Low priority } & 1 & 2 & 3 & 4 & 5 & 6 & 7 & \text { High priority }\end{array}$

2) I find the task of denture or tooth cleaning:

$\begin{array}{lllllllll}\text { Unpleasant } & 1 & 2 & 3 & 4 & 5 & 6 & 7 & \text { Pleasant } \\ \text { Boring } & 1 & 2 & 3 & 4 & 5 & 6 & 7 & \text { Exciting } \\ \text { Unenjoyable } & 1 & 2 & 3 & 4 & 5 & 6 & 7 & \text { Enjoyable }\end{array}$

3) In balancing daily duties, sufficient time is available for me to perform oral care:

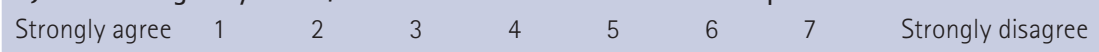

4) If the care home becomes short staffed, it is possible that oral care is not always carried out:

$\begin{array}{lllllllll}\text { Strongly agree } & 1 & 2 & 3 & 4 & 5 & 6 & 7 & \text { Strongly disagree }\end{array}$

5) Do you consider lack of resident co-operation a barrier to performing oral care?

$\begin{array}{lllllllll}\text { Not at all } & 1 & 2 & 3 & 4 & 5 & 6 & 7 & \text { Very much }\end{array}$

6) All residents in your care desire assistance with oral hygiene procedures:

$\begin{array}{llllllllll}\text { Strongly agree } & 1 & 2 & 3 & 4 & 5 & 6 & 7 & \text { Strongly disagree }\end{array}$

7) All residents in my care require assistance with denture/tooth cleaning:

$\begin{array}{llllllllll}\text { Strongly agree } & 1 & 2 & 3 & 4 & 5 & 6 & 7 & \text { Strongly disagree }\end{array}$

8) Who do you consider to be responsible for residents' oral care (please indicate all applicable options):

Staff carers $\square \quad$ Dentist $\square \quad$ Resident $\square \quad$ Resident's family $\square \quad$ Other staff member $\square$ (please indicate their title)

Training

9) Have you received any training in oral care?

Yes $\square$ (If yes proceed to Q10) No $\square$ (If no proceed to Q11)

10) Please indicate all applicable options:

Undergraduate lectures $\square \quad$ Undergraduate practical lessons $\square \quad$ In-house training $\square$

With oral health educator $\square \quad$ With dentist $\square \quad$ Other $\square$ (please indicate whom)

11) If no then please indicate why:

Absent from college/university $\square$

Too busy in the care home to attend $\square$

Not covered as part of college/university course $\square$

No training available in the care home $\square$
Absent from work $\square$

Other $\square$ (please indicate)

12) Please indicate the level of adequacy you feel with regards to your current level of training:
Very adequate $\square$
Adequate $\square$
Not sure $\square$
Inadequate $\square$
Very inadequate $\square$

13) Please indicate how confident you feel in performing oral care procedures for residents:
Very confident
23
56
$7 \quad$ Not confident

14) Please indicate how competent you feel in performing oral care procedures for residents:
Very competent 1
23
Not competent

$\begin{array}{lll}4 & 5 & 6\end{array}$

15) Please indicate how confident you feel in giving advice regarding oral care procedures to other members of staff:

$\begin{array}{lllllllll}\text { Very confident } & 1 & 2 & 3 & 4 & 5 & 6 & 7 & \text { Not confident }\end{array}$

16) Please indicate how confident you feel in giving advice regarding oral care procedures to residents:

$\begin{array}{lllllllll}\text { Very confident } & 1 & 2 & 3 & 4 & 5 & 6 & 7 & \text { Not confident }\end{array}$

17) Are you aware of the document 'Working with dependant older people to achieve good oral health' best practice statement?

Yes $\square$ (If yes please proceed to Q18) $\quad$ No $\square$ (If no please proceed to Q19)

18) Please indicate the applicable option regarding the communication of the best practise statement:

Read the document in house $\square \quad$ Discussed with colleagues $\square \quad$ Recall seeing the document but not read it $\square \quad$ Heard mention of it $\square$

19) Can you describe to me how you would care for a resident's lips?

20) Can you describe to me how you would care for a resident who has no natural teeth and dentures?

21) Can you tell me the difference between care regimes for plastic dentures and dentures with metal parts?

22) Can you describe to me how you would care for a resident's natural teeth?

23) Can you describe how you would care for the soft tissues of a resident's mouth?

24) Can you describe how you would care for a resident with a dry mouth?

25) What is the frequency of dental examination required for?

A resident with no natural teeth? $\square \quad$ A resident with some/all remaining natural teeth? $\square$

Don't know $\square$ 


\section{Appendix 3 Knowledge check points for care home $(\mathrm{N})$ staff}

Taken from 'Working with dependent older people to achieve good oral health' protocol for daily oral care, QIS (May 2005)

Applied to Questions 19-25 of Questionnaire (Appendix 2)

Q19) Care of lips

1. Clean using water-moistened gauze

2. Protect with lubricant (eg Oralbalance gel)

020) Care of the person who is edentulous (no natural teeth) with dentures

1. Dentures marked with the person's name (eg 'Identure' Denture Marking System, Geri Incorporated)

2. Dentures left out at night

3. Plastic dentures soaked in sodium hypochlorite solution

4. Dentures cleaned using individual brush under running water

5. Dentures rinsed after meals

6. Small quantity of fixative used if required

7. Fixative cleaned off and replaced before meals and cleaned off at night

021) Difference between care regimes for plastic dentures and dentures with metal parts

Dentures with metal parts should be soaked in chlorhexidine rather than sodium hypochlorite solution

022) Care of natural teeth

1. Teeth cleaned twice daily and after meals

2. Fluoride toothpaste

3. Soft toothbrush

4. Corsodyl mouthwash or spray or gel if additional plaque control required

5. Patients happy using powered toothbrush to continue with this

023) Care of oral mucosa

1. Inspect in good light

2. Report unusual appearances

3. Clean using water-moistened gauzed fingers, sponge sticks, TePe special care toothbrush or baby tooth brush

024) Care of the person with a dry mouth

1. Oral lubrication in the form of sips of water or spray or the use of mucin-based artificial saliva

2. Use of high dose fluoride toothpaste

025) Frequency of dental examination required for:

A resident with no natural teeth: minimum of every 12 months

A resident with some/all remaining natural teeth: minimum of every six months (ideally more frequently)

Recall interval should be individually assessed and determined for each patient by the dentist. 